\title{
Fever and antipyresis in infection
}

Paul J Young BSc(Hons), MB ChB, FCICM Honorary Senior Research Fellow and Project Leader

Manoj K Saxena MB BChir, FRACP, FCICM Honorary Senior Research Fellow ${ }^{2}$

Richard W Beasley MB ChB, DSc, FRACP Honorary Senior Research Fellow and Project Leader

1 Intensive Care Research Programme, Medical Research Institut of New Zealand Wellington, New Zealand

2 Division of Critical Care and Trauma, George Institute for Global Health, Sydney, NSW.

paul.young@ ccdhb.org.nz

MJA 2011; 195: 458-459 doi: 10.5694/mjall.10502 t has been known since Hippocrates' time that "progressive paralysis" due to neurosyphilis sometimes resolves after an illness associated with high fever. This observation led Julius Wagner-Jauregg to propose, in 1887, that inoculation of malaria might be a justifiable therapy for progressive paralysis. His rationale was that one could replace an untreatable condition with a treatable one malaria being treatable with quinine. In 1917, he tested his hypothesis in nine patients who had paralysis due to syphilis by injecting them with blood from patients who had malaria. Remission of paralysis occurred in three patients. This led to further study on more than a thousand patients, in which remission occurred in $30 \%$ of patients who had neurosyphilis-related progressive paralysis and were "treated" with malaria-induced fever, compared with spontaneous remission rates of only $1 \%$. This work on fever therapy led to Wagner-Jauregg being awarded the Nobel Prize in Physiology or Medicine in 1927. Even after the discovery of antibiotics rendered fever therapy for syphilis obsolete, the prevailing view 50 years ago was that fever was an important mechanism of intrinsic resistance against infectious disease.

Since then, our attitude to fever appears to have moved 180 degrees. The advice to "drink plenty of fluids and take regular paracetamol" is among the most common advice issued in ambulatory care settings. Even among the sickest patients in our intensive care units, the use of antipyretics to treat fever is ubiquitous.

The reasons for the use of antipyretics having become so common are complex. These medicines have dual effects of analgesia and antipyresis. The availability of many antipyretic medicines over the counter has led to a widely held view that they are without risk.

Evidence in support of paracetamol administration in a general practice setting comes from a randomised placebo-

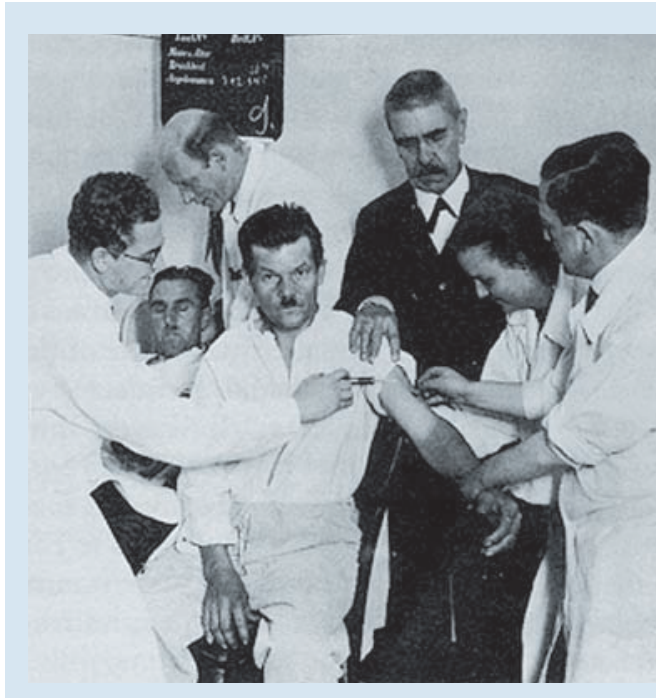

Julius Wagner-Jauregg (in black jacket) watching the transfusion of blood from a patient with malaria to a patient with neurosyphilis, to trigger fever. This approach won Wagner-Jauregg the 1927 Nobel Prize in Physiology or Medicine.

\section{Summary}

- Fever is an important mechanism of intrinsic resistance against infectious disease.

- A variety of studies point to a potential detrimental effect of temperature lowering in infectious disorders, but high-quality evidence from randomised controlled trials is lacking.

- In ambulatory care settings, we need to know whether antipyretics influence the severity and duration of illnesses and, in critically ill patients, whether antipyretics affect mortality.

controlled trial in children with viral illnesses. ${ }^{1}$ This study showed that paracetamol increased activity but not mood, comfort or appetite - the small size of the effect was highlighted by the inability of parents to correctly guess whether their children had received paracetamol or placebo. In intensive care patients, administration of ibuprofen has been shown to reduce heart rate and minute ventilation; ${ }^{2}$ proponents of antipyretic administration argue that this may be important in critically ill patients with limited cardiac or respiratory reserve.

These considerations need to be balanced against the numerous studies which suggest that administration of antipyretics has the potential to adversely affect the course of infectious disorders. In adults, administration of antipyretics increases rhinovirus shedding and worsens nasal symptoms in patients with the common cold. ${ }^{3}$ Similarly, in children with varicella infection, paracetamol administration increases the duration of crusting of chickenpox lesions. ${ }^{4}$ Furthermore, a recent randomised controlled trial in children receiving vaccination showed that administration of paracetamol leads to a blunted immunological response to the vaccine. ${ }^{5}$

To date, only one randomised controlled trial has addressed the impact of different paracetamol-based antipyretic therapy strategies on the development of infectious complications in intensive care. ${ }^{6}$ This trial was stopped by a data safety monitoring board because of a trend towards an increased number of deaths due to sepsis in patients in the aggressive antipyretic treatment group. ${ }^{6}$ In contrast, a large placebo-controlled trial of ibuprofen in sepsis did not show a significant effect on mortality. ${ }^{2}$ However, this trial was not designed to investigate the use of ibuprofen as an antipyretic, and the anti-inflammatory actions of ibuprofen and concomitant use of paracetamol confound interpretation.

In support of the potential detrimental effect of lowering temperature, a laboratory study involving 432 strains of bacteria and 17 antimicrobials demonstrated that virtually all bacterial strains exhibited increasing antimicrobial susceptibility with increasing temperature within the physiological febrile range. ${ }^{7}$ Some strains of bacteria reduce their replication rate as temperature rises and even die at temperatures within the physiological febrile range. ${ }^{8}$ In the cases of influenza and Streptococcus pneumoniae infection, animal data suggest that the temperature sensitivity of strains in vivo is related to their virulence in the host: more 
temperature-resistant strains cause more severe disease. ${ }^{9,10}$ Suppression of the febrile response to infection with antipyretic therapy has been shown to increase the risk of mortality arising from viral, bacterial and parasitic infections in several mammalian species. ${ }^{11}$

Parents of young children have observed that paracetamol has a remarkable calming effect when administered to an unsettled child. ${ }^{12}$ Furthermore, parents often administer paracetamol for its antipyretic effect to children with minimal or no fever, ${ }^{13}$ and perceived parental pressure is a significant factor in determining whether nurses administer paracetamol to paediatric patients. ${ }^{14}$ The recent determination that paracetamol exerts its analgesic effects through indirect stimulation of cannabinoid receptors ${ }^{15}$ puts a new perspective on the calming effects that parents have observed. It is interesting to consider whether, if the history of development of paracetamol as a medicine had been different, and its mechanism of action had been known from the outset, this calming effect in children would have been viewed in such a positive light.

The data presented here provide "proof of concept" that the current dogma needs to be questioned. However, we lack high-quality evidence on which to base clinical practice. Randomised controlled clinical trials evaluating the effects of antipyretics are needed. In ambulatory care settings, we need to know whether antipyretics influence the severity and duration of illnesses and, in the most critically ill, we need to know whether antipyretics affect mortality.

Competing interests: Richard Beasley has received consultancy fees from GlaxoSmithKline and grants from AstraZeneca, Novartis and Chiesi.

Provenance: Commissioned; externally peer reviewed.
1 Kramer MS, Naimark LE, Roberts-Bräuer R, et al. Risks and benefits of paracetamol antipyresis in young children with fever of presumed viral origin. Lancet 1991; 337: 591-594.

2 Bernard G, Wheeler A, Russell J, et al. The effects of ibuprofen on the physiology and survival of patients with sepsis. N Engl J Med 1997; 336: 912919.

3 Graham NM, Burrell CJ, Douglas RM, et al. Adverse effects of aspirin, acetaminophen, and ibuprofen on immune function, viral shedding, and clinical status in rhinovirus-infected volunteers. J Infect Dis 1990; 162: 12771282.

4 Doran TF, De Angelis C, Baumgardner RA, Mellits ED. Acetaminophen: more harm than good for chickenpox? J Pediatr 1989; 114: 1045-1048.

5 Prymula R, Siegrist CA, Chlibek R, et al. Effect of prophylactic paracetamol administration at time of vaccination on febrile reactions and antibody responses in children: two open-label, randomised controlled trials. Lancet 2009; 374: 1339-1350.

6 Schulman Cl, Namias N, Doherty J, et al. The effect of antipyretic therapy upon outcomes in critically ill patients: a randomized, prospective study. Surg Infect (Larchmt) 2005; 6: 369-375.

7 Mackowiak PA, Marling-Cason M, Cohen RL. Effects of temperature on antimicrobial susceptibility of bacteria. J Infect Dis 1982; 145: 550-553.

8 Mackowiak PA, Ruderman AE, Martin RM, et al. Effects of physiologic variations in temperature on the rate of antibiotic-induced bacterial killing. Am JClin Pathol 1981; 76: 57-62.

9 Oxford JS, Corcoran T, Schild GC. Naturally occurring temperature-sensitive influenza a viruses of the HIN1 and H3N2 subtypes. J Gen Virol 1980; 48: 383389.

10 Enders JF, Shaffer MF. Studies on natural immunity to Pneumococcus Type III. I. The capacity of strains of Pneumococcus Type III to grow at $40^{\circ} \mathrm{C}$ and their virulence for rabbits. J Exp Med 1936; 64: 7-18.

11 Kluger MJ, Kozak W, Conn CA, et al. The adaptive value of fever. Infect Dis Clin North Am 1996; 10: 1-22.

12 Lagerløv P, Helseth S, Holager T. Childhood illnesses and the use of paracetamol (acetaminophen): a qualitative study of parents' management of common childhood illnesses. Fam Pract 2003; 20: 717-723.

13 Sullivan JE, Farrar HC. Fever and antipyretic use in children. Pediatrics 2011; 127: 580-587.

14 Walsh AM, Edwards HE, Courtney MD, et al. Fever management: paediatric nurses' knowledge, attitudes and influencing factors. J Adv Nurs 2005; 49: 453-464.

15 Bertolini A, Ferrari A, Ottani A, et al. Paracetamol: new vistas of an old drug. CNS Drug Rev 2006; 12: 250-275. 